\title{
Estudo da eficácia e tolerabilidade do salmeterol comparativamente ao salbutamol em pacientes com asma brônquica
}

\author{
M.A.de Oliveira, J .R. DE Brito J ARdiM, S.M. FARESIN, S.R.R. LuCAS, L.E. NeRY \\ Disciplina de Pneumologia - U niversidade F ederal de São Paulo, São Paulo, SP.
}

RESUMO - Os $\beta_{2}$-agonistas constituem um dos pilares do tratamento da asma brônquica, porém sua curta duração de ação exige uso freqüente e a associação com outras drogas broncodilatadoras. O surgimento dos $\beta_{2}$-agonistas de longa ação pode representar um avanço na terapêutica da asma brônquica.

Овј етіvo. O presente estudo propõe-se a avaliar, em nosso meio, a eficácia e a tolerabilidade do salmeterol (SM), comparativamente ao salbutamol (SB), em pacientes com asma leve e moderada.

Métodos. Após uma etapa de estabilização de duas semanas, os pacientes utilizaram salmeterol $50 \mathrm{mcg}$ duas vezes ao dia, e salbutamol $200 \mathrm{mcg}$ quatro vezes ao dia, durante o período de quatro semanas, seguindo um esquema duplo cego, aleatório, de grupos paralelos. Foram estudados 60 pacientes que preencheram os seguintes critérios de inclusão: VE $F_{1}$ (Volume Expiratório Forçado no 1o segundo) $>50 \%$; variação diurna do PFE (Pico do Fluxo Expiratório) $>15 \%$ ou resposta do VE $F_{1}$ ao $B D>15 \%$, gradação de sintomas $>2$ (escala de 0 a 5 ) em quatro dos últimos sete dias.

Resultados. Dos 60 pacientes estudados, sete foram excluídos no período de tratamento (ver Métodos), sendo concluído o estudo com 25 pacientes no grupo salmeterol e 28 no grupo salbu- tamol. No período de estabilização, não houve diferença significante entre os grupos, compa-

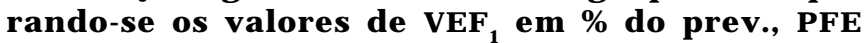
matinal, gradação de sintomas e gravidade da asma. $O$ percentual de melhora do VEF $F_{1}$ do PFE matinal nos pacientes que receberam salmeterol foi significantemente mais elevado entre 2 e 4 semanas de tratamento, em relação aos pacientes que receberam salbutamol $(p<0,05)$. Da mesma forma, o grupo salmeterol apresentou redução significante nos valores médios dos sintomas no período noturno na 1o quinzena de tratamento. Em relação ao número de inalações de socorro utilizadas, efeitos colaterais, freqüência cardíaca, pressão arterial sistêmica e dosagem de potássio, não houve diferença significante entre os grupos.

Conclusão. Este estudo demonstrou que, em pacientes com asma leve e moderada, o salmeterol na dose de $100 \mathrm{mcg} / \mathrm{di}$ a elevou o VEF , O PFE matinal e apresentou diminuição significantemente maior dos sintomas noturnos em relação aos observados no grupo salbutamol, e que a tolerância aos medicamentos estudados foi semeIhante nos dois grupos.

Unitermos: Asma. Salmeterol - tratamento.

\section{NTR ODUÇÃO}

Nos últimos 20 anos, houve um significativo avanço no conhecimento da fisiopatologia da asma brônquica, reconhecida atualmente como uma doença inflamatória ${ }^{1}$, o que alterou o enfoque do seu tratamento. Drogas antiinflamatórias são utilizadas para atuar no mecani smo etiopatogênico básico da doença e drogas broncodilatadoras, no alívio imediato do sintoma, através do relaxamento da musculatura lisa. Os $\beta_{2}$-agonistas têm sido o tratamento de escolha para a crise aguda da asma e também são utilizados de forma regular no tratamento da asma, apesar de controvérsias existentes na literatura2,5. Quando utilizados de forma reguIar, os $\beta_{2}$-agonistas convencionais têm a desvantagem de apresentar curta duração de ação ${ }^{6}$, exigindo o uso freqüente e a associação de outras drogas broncodilatadoras, principalmente em pacientes com asma noturna, não controlados por antiinflamatórios.

O xinafoato de salmeterol é um $\beta_{2}$-agonista de longa duração, cuja ação prolongada tem sido atribuída, principalmente, a interações físicoquímicas desse fármaco com a membrana bipolar do músculo li so 7 . Vários estudos clíni cos têm comprovado a eficácia do sal meterol como droga broncodilatadora protegendo contra a hiper-responsividade provocada pela histamina, metacolina, exercício e ar frio ${ }^{8-11}$. É administrado por via inalatória, produzindo broncodilatação por 12 horas ${ }^{12}$, sendo referidos efeitos colaterais comuns aos $\beta_{2}$-agonistas convencionais ${ }^{13}$. Trabal hos clínicos tềm demonstrado que o salmeterol promove uma melhora global da asma brônquica, com meIhora da função pulmonar, diminuição dos sinto- 


\begin{tabular}{|c|c|}
\hline \multicolumn{2}{|l|}{ Quadro - Gradação de sintomas do período noturno e diurno } \\
\hline Período noturno & Período diurno \\
\hline $0=$ Sem sintomas durante a noite & $0=$ Sem sintomas durante $\mathrm{o}$ dia \\
\hline 1 = Sintomas que o despertem uma vez durante a noite ou que & $1=$ Sintomas em um pequeno período durante o dia \\
\hline façam período durante o dia despertar mais cedo pela manhã & 2 = Sintomas em dois ou mais pequenos períodos durante o dia \\
\hline $\begin{aligned} 2= & \text { Sintomas que o despertem duas ou mais vezes à noite } \\
& \text { (inclusive despertar mais cedo) }\end{aligned}$ & $\begin{array}{l}3=\text { Sintomas durante a maior parte do dia afetando a atividade } \\
\text { diária normal }\end{array}$ \\
\hline $3=$ Sintomas que o mantenham acordado a maior parte da noite & $4=$ Sintomas de tal gravidade que impeçam o comparecimento ou \\
\hline 4 = Os sintomas são tão graves que o impedem de dormir & desenvolvimento de atividades diárias normais \\
\hline
\end{tabular}

mas noturnos e diurnos e redução da utilização da medicação de socorro ${ }^{13,14}$.

O propósito de nosso estudo foi avaliar a eficácia e tol erabilidade do sal meter ol $50 \mathrm{mcg}$ duas vezes ao dia, comparativamente ao salbutamol, 200mcg quatro vezes ao dia, no tratamento de pacientes com asma leve e moderada durante período de quatro semanas. Nossa hipótese é de que o salmeterol, um $\beta_{2}$-agonista de longa ação, produz um mel hor controle da asma, sem aumento da incidência de efeitos colaterais em relação ao sal butamol, um $\beta_{2}$-agonista de curta ação.

\section{MATERIAL E MÉTODOS}

O diagnóstico de asma baseou-se na história clínica, com os pacientes classificados como tendo asma leve a moderada, de acordo com o consenso internacional ${ }^{15}$, e preenchendo os seguintes critérios de inclusão: gradação de sintomas $>2$ (quadro acima), $V E F_{1}$ (volume expiratório forçado no 1을 segundo) $>50 \%$ do previsto e reversibilidade do $\mathrm{VEF}_{1}>15 \%$ após 15 minutos da inalação de $200 \mathrm{mcg}$ de salbutamol ou uma variação diurna do pico do fluxo expirátorio (PFE) de pelo menos $15 \%$ em 4 dos últimos 7 dias, calculada da seguinte maneira:

$$
\text { PFE }=\frac{\text { PFE noturno }- \text { PFE manhã }}{\text { PFE noturno }} \times 100 \%
$$

De 136 pacientes avaliados que preencheram os critérios iniciais de inclusão, 76 foram excluídos durante a fase de estabilização por não apresentar os critérios referentes à função pulmonar e/ou cooperação ao protocolo, permanecendo, então, 60 pacientes que foram informados dos detalhes e objeti vos do estudo e cujo consentimento foi obtido por escrito. Dos pacientes selecionados, 43 eram do sexo feminino e 17 do masculino (idade entre 18 e 64 anos, com média de 37 anos).

Após uma etapa de estabilização de duas semanas, os pacientes utilizaram salmeterol $50 \mathrm{mcg}$, duas vezes ao dia (6-7h; 22-23h), mais placebo, duas vezes ao dia (11-12h; 17-18h), ou sal butamol 200mcg, quatro vezes ao dia, seguindo um esquema duplo cego, al eatório, de grupos paralelos. Os pacientes utilizaram salbutamol como medicação de socorro, e os corticosterói des orais (até $20 \mathrm{mg}$ de prednisona/dia) e inalatórios, quando previamente utilizados, foram mantidos; outros $\beta_{2}$-agonistas, xantinas e anticolinérgicos foram suspensos. $O$ uso de $\beta_{2}$-agonistas (diferente das drogas em estudo), metilxantinas, corticosteroídes e anticolinérgicos foram permitidos nas exarcebações agudas da asma. Os pacientes que necessitaram de tratamento de manutenção com estas drogas e tiveram necessidade de dose superior a $20 \mathrm{mg} / \mathrm{dia}$ de esteróides, por período superior a três dias, foram retirados do estudo. E m cada consulta eram anotados dados antropométricos e clínicos dos pacientes, incluindo pressão arterial sistêmica e freqüência cardíaca em repouso. Os pacientes recebiam um registro diário padronizado, sendo orientados a anotar, pela manhã e à noite, a gradação dos sintomas (escala de 0 a 5 - ver quadro), o número de inalações de socorro utilizadas e três medidas consecutivas do PFE com um aparelho mini-wright, antes da inalação da droga em estudo, sendo considerada a medida de maior valor. Espirometrias pré e pós-broncodilatador foram real izadas com o objetivo de preencher os critérios de seleção e servir como parâmetro de função ventilatória basal do paciente e repetidas em cada consulta da etapa de tratamento, em horário próximo às anteriores (9-10h). O aparel ho utilizado foi o Diagnostic System Med Graphics MCG-CPX ${ }^{16}$. $\mathrm{Na}$ etapa de estabilização e no término do tratamento, foram efetuadas el etrocardiogramas de 12 derivações e coleta de sangue para realização de exames hematológicos e bioquímicos.

A eficácia do tratamento foi analisada através das seguintes variáveis: gradação de sintomas, número de inalações de socorro utilizadas, pico do fluxo expiratório e espi rometria e variação diurna do PFE (calculada no período de sete dias). Em relação a tolerabilidade, foram analisados: efeitos 


\begin{tabular}{|c|c|c|}
\hline \multicolumn{3}{|c|}{$\begin{array}{l}\text { Tabela } 1 \text { - Características clínicas e antropométricas do } \\
\text { pacientes do grupo salmeterol e salbutamol }\end{array}$} \\
\hline & Salmeterol & Salbutamol \\
\hline No de pacientes & 25 & 28 \\
\hline \multicolumn{3}{|l|}{ Sexo } \\
\hline Masculino (n) & 7 & 9 \\
\hline Feminino $(n)$ & 18 & 19 \\
\hline Idade (anos) ${ }^{*}$ & 32,5 & 42,4 \\
\hline Altura $(\mathrm{cm})^{*}$ & 157,7 & 156,1 \\
\hline Peso $(\mathrm{kg})^{*}$ & 62,7 & 65,5 \\
\hline Duração da asma (anos)* & 16,9 & 19,5 \\
\hline \multicolumn{3}{|l|}{ Tabagismo (n) } \\
\hline Não & 21 & 22 \\
\hline Sim & - & 1 \\
\hline Ex & 4 & 5 \\
\hline \multicolumn{3}{|l|}{ Gravidade da asma (n) } \\
\hline Leve & 11 & 13 \\
\hline Moderada & 14 & 15 \\
\hline
\end{tabular}

colaterais relacionados a medicação em estudo (avaliação de segurança), análise da freqüência cardíaca e pressão arterial sistêmica e das alterações eletrocardiográficas e bioquímicas, especificamente, dosagem de potássio.

\section{ANÁLISE ESTATÍSTICA}

Para comparar os períodos pré (estabilização), $\mathrm{T}_{1}$ e $\mathrm{T}_{2}$ (respectivamente $1^{\text {a }}$ e $2^{\mathrm{a}}$ quinzena de tratamento), em relação às variáveis espirométricas, gradação de sintomas, número de inalações de socorro utilizadas e PFE, foi utilizada a análise de variância por postos de Friedman ${ }^{17}$, e quando mostrou diferença significante foi complementada pel o teste de comparações múltiplas ${ }^{18}$. Utilizamos o teste de Mann-Whitney para duas amostras independentes, a fim de comparar os dois grupos em relação às variáveis analisadas. O teste de Wilcoxon para dados pareados foi utilizado para comparar os períodos pré e pós-tratamento em relação aos valores de potássio. As diferenças percentuais (DT) das etapas consideradas foram cal culadas pela seguinte fórmula:

$$
\mathrm{DT}=\mathrm{E}_{\mathrm{tapa}} \mathrm{T}_{1} \frac{\text { ou } \mathrm{T}_{2}}{\mathrm{E}}-\text { Etapa pré } \times 100
$$

Em todos os testes, fixou-se em 0,05 ou $5 \%$ o nível de rejeição da hipótese de nulidade, assinalando-se com um asterisco os val ores significantes.

\section{RESULTADOS}

Dos 60 pacientes incluídos no protocolo, sete pacientes foram excluídos durante a etapa de tratamento. Do grupo salmeterol (SM), dois pacientes



口 SALBUTAMOL I SALMETEROL

Gráfico 1 - Valores da variação percentual DT $=(T$ PRÉ/ PRÉ em \%) do volume expiratório forçado no 1o segundo (VEF ) em\% do previsto, aos 15 dias (T 1) e aos 30 dias (T2), dos pacientes com asma recebendo salmeterol e salbutamo $* p<0,05$.

foram excluídos devido à crise de asma, dois por não aderência ao tratamento e um paciente, por efeito colateral (tremores e pal pitação) atribuídos ao medicamento. Do grupo salbutamol (SB), dois pacientes foram excluídos devido à crise de asma. Dos 53 pacientes analisáveis, 25 eram do grupo salmeterol e 28 do grupo salbutamol. Do ponto de vista clínico, os pacientes de ambos os grupos tinham duração e gravidade da asma brônquica semel hantes (tabela 1). Em relação a corticoterapia, $56 \%$ dos pacientes do grupo salmeterol e $42 \%$ do grupo sal butamol faziam uso de corticosteroídes inalatório e/ou orais.

$\mathrm{N}$ a etapa de estabilização, não houve diferença significante entre os grupos comparando-se os valores de $V E F_{1}$ em \% prev., PFE matinal e noturno, gradação de sintomas e número de inalações de socorro utilizadas. Em relação a variação diurna do PFE, o grupo salmeterol apresentou uma variabilidade significantemente maior do que o grupo sabutamol.

\section{Espirometria}

$\mathrm{Na}$ etapa de tratamento, o percentual de melhora do VEF $F_{1}$ matinal pré-broncodilatador dos pacientes que receberam salmeterol foi significantemente mais elevado $(p<0,05)$ em relação aos pacientes que receberam salbutamol com duas (SM= $15,2 \%$; $S B=2,3 \%$ ) e quatro semanas ( $S M=16,6 \%$; $\mathrm{SB}=1,8 \%$ ) de tratamento (gráfico 1). Os valores de $\mathrm{CVF}, \mathrm{FEF}_{25-75 \%}$ e $\mathrm{FEF}_{50 \%}$ apresentaram comportamento semelhante ao $V E F_{1}$, evidenciando melhora significante no grupo salmeterol em relação ao salbutamol. 


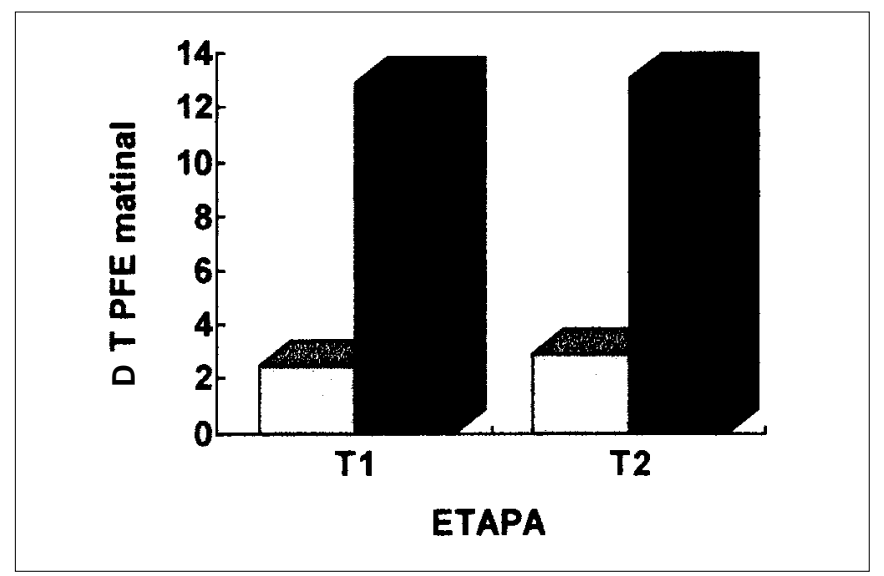

Gráfico 2 - Valores médios da variação percentual $\mathrm{DT}=$ (T-PRÉ/PRÉ em \%) do pico do fluxo expiratório matinal com 15 dias (T1) e 30 dias do tratamento (T2), dos pacientes com asma recebendo salmeterol e salbutamol $* p<0,05$.

\section{Pico do fluxo expiratório}

$\mathrm{Na}$ etapa de estabilização, os valores médios do PFE matinal foram 328L/min para o grupo salmeterol e $311 \mathrm{~L} / \mathrm{min}$ para o grupo salbutamol. No horário noturno, os valores médios foram, respectivamente, $344 \mathrm{~L} / \mathrm{min}$ e $330 \mathrm{~L} / \mathrm{min}$. Na etapa de tratamento, houve aumento significante $(p<0,05)$ nos valores médios, tanto do PFE matinal como do noturno, no grupo salmeterol, o mesmo não ocorrendo com o sal butamol. E m rel ação ao percentual de melhora (DPFE), observamos que no horário matinal houve um aumento significante do PFE durante o período de tratamento dos pacientes que fizeram uso do salmeterol (gráfico 2). No grupo salbutamol, não observamos diferença significante entre a etapa de estabilização e a de tratamento, tanto no horário matinal como no noturno.

Quando analisamos a variação diurna do PFE, observamos que no grupo salmeterol houve significante redução desta variação quando comparamos a etapa de estabilização com a $1^{\text {a }}\left(T_{1}\right)$ e $2^{\text {a }}\left(T_{2}\right)$ quinzena de tratamento (pré=18,5; $\mathrm{T}_{1}=11,7 ; \mathrm{T}_{2}=$ $12,4)$. Em relação ao grupo sal butamol, não observamos diferença significante entre a etapa de estabilização e de tratamento (pré=13,7; $T_{1}=11,93$; $\mathrm{T}_{2}=11$ ).

\section{Gradação de sintomas}

$\mathrm{Na}$ etapa de tratamento, os valores médios dos sintomas do período diurno, tanto no grupo salmeterol como no salbutamol, foram significantemente menores em relação aos valores da etapa de estabilização, sendo esta redução mais pronuncia-

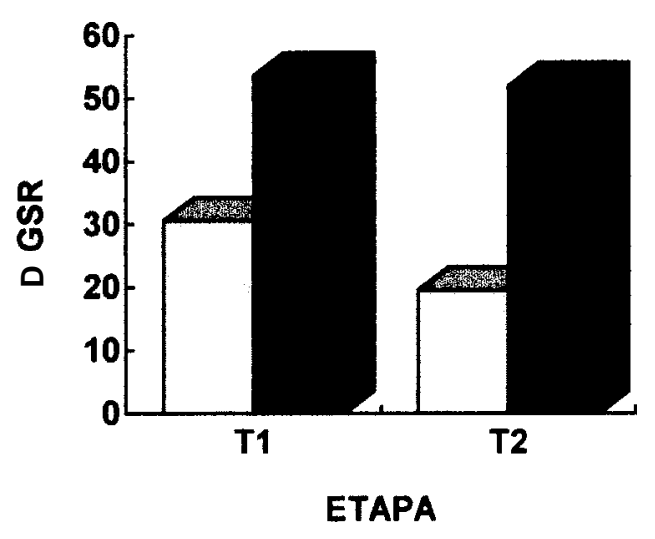

Gráfico 3 - Valores médios da variação percentual DT=(T-PRÉ/ PRÉ em \%) da gradação de sintomas respiratórios (GSR) do período noturno com 15 dias (T1) e 30 dias do tratamento (T2), dos pacientes com asma recebendo salmeterol e salbutamol $*=p<0,05$.

da, embora não significante no grupo salmeterol. Em relação ao período noturno, tanto o grupo salmeterol como o salbutamol, apresentaram redução significante nos valores médios dos sintomas em relação à etapa de estabilização. Quando comparamos a gradação de sintomas do grupo salmeterol (pré $=0,73 ; T_{1}=0,33 ; T_{2}=0,33$ ) com a do grupo sabutamol (pré=1,04; $\mathrm{T}_{1}=0,70 ; \mathrm{T}_{2}=0,66$ ) observamos, na primeira quinzena, uma diferença signifi cante $(p<0,05)$ na redução dos valores médios dos sintomas do grupo salmeterol em relação ao grupo salbutamol ( $\mathrm{SM}=53,6$ vs. $\mathrm{SB}=30,6$ ) (gráfico 3). Na segunda quinzena, esta diferença, embora acentuada, como evidenciado pela variação percentual de melhora dos pacientes do grupo salmeterol ( $S M=-52$ vs. $S B=-19,5)$, não foi significante. Em relação ao pós-tratamento, os valores médios dos sintomas, tanto do grupo salmeterol como do sal butamol, retornaram a valores próximos à etapa de estabilização.

\section{Número de inalações de socorro utilizadas}

Durante o tratamento, no período diurno, tanto o grupo salmeterol (pré $=1,10 ; \mathrm{T}_{1}=0,39 ; \mathrm{T}_{2}=0,23$ ) como o grupo sal butamol (pré=1,34; $T_{1}=0,70 ; T_{2}^{2}=0,68$ ), apresentaram valores médios significantemente menores em relação à etapa de estabilização. No período noturno, da mesma forma, os dois grupos apresentaram redução significante nos valores médios do número de inalações utilizadas em relação à etapa de estabilização. Esta redução foi maior (mas não si gnificante) no grupo salmeterol. Em relação ao pós-tratamento, os valores médios do número de inalações de socorro utilizadas, tan- 
Tabela 2-Efeitos colaterais apresentados pelos pacientes do grupo salmeterol e salbutamol durante a etapa de tratamento

\begin{tabular}{|lccc|}
\hline & Salmeterol & Salbutamol & Placebo \\
Efeito colateral & $№$ & $№$ & № \\
Tremor & 2 & 2 & - \\
Palpitação & 1 & 1 & - \\
Cefaléia & 1 & - & - \\
Boca seca & 2 & 1 & - \\
Mal estar & - & - & 1 \\
Tontura & - & - & 1 \\
Insônia & 1 & 1 & - \\
\hline *Placebo utilizado no grupo salmeterol & & \\
\hline
\end{tabular}

to no grupo salmeterol como no salbutamol, retornaram a valores próxi mos à etapa de estabilização.

\section{Efeitos colaterais}

Durante o tratamento, a incidência global de efeitos colaterais relacionados às medicações em estudo relatadas pelos pacientes foi de 25,9\%. Foram relatados palpitação, cefaléia, boca seca e insônia, tanto no grupo salmeterol como no salbutamol (tabela 2).

Em relação à freqüência cardíaca e pressão arterial sistêmica, não houve diferença significante, tanto no grupo salmeterol como no salbutamol, quando comparado a etapa de estabilização com o tratamento; e nem apresentaram diferença significante entre si aos 15 dias $\left(T_{1}\right)$ e aos 30 dias $\left(\mathrm{T}_{2}\right)$ de tratamento.

E $m$ relação aos registros el etrocardiográficos e dosagem de potássio realizadas antes e após o tratamento, não observamos variação significante, tanto no grupo salmeterol como no sal butamol.

\section{DISCUSSÃO}

O salmeter ol e o sal butamol, como já comentado, são drogas $\beta_{2-a}$ drenérgicas com diferenças na duração de ação. Enquanto o salmeterol promove broncodilatação por um período de 12 horas, o salbutamol tem efeito broncodilatador até 6 de horas. O nosso protocolo, buscando demonstrar esta diferença, realizou as espirometrias com o intervalo maior que 6 horas da utilização das drogas em estudo. Em relação à espirometria, os pacientes do grupo sal meterol apresentaram aumento significante nos valores de todas as variáveis analisadas, confirmando estudos anteriores, que mostraram o efeito broncodilatador prolongado do salmeterol. Em rel ação ao PFE, os val ores médios do horário noturno foram mais elevados em ambos os grupos em relação ao horário matinal, o que é compatível com o padrão circadiano apresentado pel os pacientes portadores de asma brônquica com uma variação que pode chegar a $50 \%{ }^{19}$. Na etapa de tratamento, os valores médios do PFE dos pacientes do grupo salmeterol em relação ao salbutamol foram significantemente mais el evados, tanto no horário matinal como no noturno. Quando comparamos as duas drogas, observamos que, no horário matinal, o percentual de melhora do grupo salmeterol foi significantemente maior do que o salbutamol, o que não foi observado no horário noturno. Achados semelhantes são descritos por outros autores ${ }^{13}$. A melhora do PFE matinal reflete redução da broncoconstrição noturna pel o sal meterol, o que significa melhor controle dos pacientes portadores de asma noturna, corroborando os achados de outros autores que demonstraram uma mel hora objetiva na qualidade do sono de pacientes portadores de asma noturna ${ }^{20}$ e também mel hora do padrão do sono através de um questionário de qualidade de vida $^{21}$. Em relação à variação do PFE, apesar da escolha al eatória dos pacientes, o grupo salmeterol apresentou-a maior na etapa de estabilização em relação ao grupo salbutamol, o que prejudicou a comparação entre os grupos, porém não invalida o fato que, em relação à etapa de estabilização, o grupo salmeterol apresentou redução desta variação, o mesmo não se observando no grupo salbutamol. Outros estudos demonstram que o salmeterol reduz a variação do PFE, quando comparado ao salbutamol ${ }^{13}$.

Em nosso estudo, o grupo salmeterol apresentou redução significativa dos sintomas respiratórios, comparativamente ao sal butamol; entretanto somente no período noturno da primeira quinzena é que os valores médios foram significantes (gráfico 3). Nos outros períodos, a avaliação estatística mostrou comportamento marginal em relação à significância estatística para o grupo salmeterol. A redução dos sintomas apresentada pelo grupo salmeterol é particularmente importante para os pacientes portadores de asma noturna, como já demonstrada por outros estudos que compararam salmeterol com placebo ${ }^{20}$ e com teofilina de liberação lenta associado ao cetotifeno na asma noturna ${ }^{22}$. E m rel ação ao número de inalações de socorro utilizadas, ambos os grupos apresentaram redução significante dos valor es médios durante a etapa de tratamento, entretanto, não diferindo significantemente entre si, ao contrário do referido em outro estudo ${ }^{13}$, que demonstrou redução significante do número de inalações de socorro utilizadas, tanto durante o período diurno como no noturno no grupo salmeterol, em relação ao grupo salbutamol. 
Tanto o grupo salmeterol como o salbutamol não apresentaram variação significativa da freqüência cardíaca durante a etapa de tratamento, o que está de acordo com outros estudos, que demonstraram que a dose de $50 \mathrm{mcg}$ de salmeterol produziu efeito semel hante ao salbutamol sobre a freqüência cardíaca e que a el evação da dose do salmeterol para $200 \mathrm{mcg}$ produzia aumento da freqüência cardíaca com efeito máximo depois de duas horas ${ }^{12}$. A pressão arterial sistêmica de ambos os grupos em nosso estudo também não apresentou variação significante na etapa de tratamento em relação aos valores da etapa de estabilização. Achados semelhantes foram observados por outros autores ${ }^{23}$.

Ambos os grupos apresentaram efeitos colaterais semelhantes e já esperados para $\beta_{2}$-agonistas. Dos efeitos colaterais citados, boca seca, não foi relatado por quaisquer dos estudos ${ }^{13,23}$. Outros estudos já demonstraram que a dose de 50 mcg ao dia é bem tolerada pelos pacientes, e a incidência de efeitos colaterais com o salmeterol aumenta quando os pacientes utilizam a dose de $100 \mathrm{mcg}^{23}$.

A utilização dos $\beta_{2}$-agonistas é um dos pilares do tratamento da asma brônquica desde $1969^{24}$, quando foi descrito o primeiro $\beta_{2}$-agonista sel etivo, porém o uso de $\beta_{2}$-agonista de curta ação de forma regular tem sido motivo de controvérsias, tanto pelas evi dências de associ ação com aumento da mortalidade ${ }^{25}$, como pel o aumento da hiper responsividade brônquica associada a deterioração do controle clínico da asma ${ }^{2,3}$. O salmeterol, $\beta_{2}$-agonista de longa ação, ainda não tem seu papel definido no tratamento da asma brônquica. Nosso estudo mostra, entretanto, que, após avaliação dos riscos e benefícios da utilização de um $\beta_{2}$-agonista, torna-se evidente que o salmeterol, comparativamente ao salbutamol, apresenta efeitos colaterais similares; é uma opção terapêutica valiosa, principalmente para pacientes com necessidade de uso freqüente de broncodilatadores e para aqueles portadores de asma noturna que necessitam de uma droga com ação suficiente para proporcionar alívio dos sintomas respiratórios durante uma noite de sono.

Este trabalho, parcialmente subsidiado pelo CNPq, FAPESP e Glaxo-Wellcome é a avaliação brasileira do estudo multicêntrico realizado com o salmeterol na América Latina. É parte da tese de Mestrado da Dra Maria Alenita de Oliveira.

\section{SUMMARY}

Efficacy and safety of inhaled salmeterol compared to salbutamol in patients with mild-to-moderate asthma

$\mathrm{B}_{2}$-agonists are considered one of the cornerstones of the asthma therapy, but their short action requires frequent administration and an association with other broncodilators. The development of Iong-acting $\beta 2$-agonists may represent an important improvement in asthma treatment.

Purpose. The present study was designed to assess the efficacy and safety of inhal ed salmeterol compared to salbutamol in patients with mild-tomoderate asthma.

Methods. After the two run-in weeks, the pati ents received either sal meterol 50mg twice a day or sal butamol $200 \mathrm{mg}$ four times a day, over a four week period, following a double blind, parallel group study. Sixty patients had the following inclusion criteria: $\mathrm{FEV}_{1}>50 \%$ or PEFR over the past seven days $>50 \%$ of predicted normal; reversibility of $\mathrm{FEV}_{1}>15 \%$; symptoms scores $>2$ (score 0 and 5 ) in 4 of the last seven days or PEFR variation $>15 \%$.

Results. Seven patients discontinued the protocol (see methods). Of the 53 analyzable patients, 25 were of the salmeterol group and 28 of the salbutamol group. Our results showed that in the run-in period there were not differences among the groups comparing the values of $\mathrm{FEV}_{1}$ in \% predicted, morning PEFR and asthma symptoms scores. The improvement rate of morning FEV $\mathrm{V}_{1}$ and PEFR in patients who recei ved salmeterol was significantly higher $(p<0.05)$ compared to the patients who received salbutamol, for two and four weeks of treatment. Also, the salmeterol group have shown reduction of the symptoms in the nocturnal period(significantly in the first fortnight of treatment) demonstrated by the significative increase in the symptoms improvement rate when compared salmeterol and to salbutamol groups. The number of rescue medication inhaled, side effects, heart rate, blood pressure, serum potassium dosage and electrocardiograms, did no show significative differences between the groups.

Conclusion.This study showed that in mild to moderate asthmatic patients, salmeterol in the dosage of $100 \mathrm{mg} /$ day raised the FEV 1 and the morning PEF and led to pronounced decrease in the nocturnal symptoms as compared to salbutamol. The side effects were similar. [Rev Ass Med Brasil 1998; 44(3): 169-75]

KEY WORDS: Asthma - Long-acting $\beta_{2}$-agonistssalmeterol. 


\section{REFERÊNCIAS BIBLIOGRÁFICAS}

1. Barnes PJ. Asthma. British Medical Bulletin. London, Churchill Livingstone. 1992; 48: 1.

2. Sears MR, Taylor DR., Print.CG et al. Regular inhaled betaagonist treatment in bronchial asthma. Lancet 1990; 336: 1.391-6.

3. Taylor DR, Sears MR, Herbison GP et al. Regular inhaled ? agonist in asthma: effects on exarcerbations and lung function. Thorax 1993; 48: 134-8.

4. Twentyman OP, Higenbottam TW. Controversies in respiratory medicine:regular inhaled $\beta_{2}$-agonists-clear clinical benefit or a hazard to health?- (1) $\beta_{2}$-agonists can be safely and beneficially in asthma. Respir Med 1992; 86: 471-6.

5. Page $C$, Costello J . Controversies in respiratory medicine: regular inhal ed $\beta_{2}$-agonists-clear clinical benefit or a hazard to health?- (2) Why $\beta_{2}$-agonists shoud not be used regularly. Respir Med 1992; 86: 477-9.

6. Walker SR, Evans ME, Richards AJ et al. The clinical pharmacology of oral and inhaled salbutamol. Clin Pharmacol Ther 1972; 13(6): 861-7.

7. Anderson GP, Linden A, Rabe KF. Why are long-acting beta-adrenoceptor agonists long acting? E ur Respir J 1994; 7: 569-78.

8. Gongora HC, Wisniewski AFZ, Tattersfied AE. A single-dose comparison of inhaled albuterol and two formulations of salmeterol on airway reactivity in asthmatic subjects. Am Rev Respir Dis 1991; 144: 626-9.

9. Deron EY, Pauwels RA, Van Der Stracten MEF. The effect of inhaled salmeterol on methacholine responsiveness in subjects wich asthma up to 12 hours. J Allergy Clin Immunol 1992; 89: 811-5.

10. Anderson SD, Rodwell LT, Du Toit J et al. Duration of protection by inhaled salmeterol in exercise-induced asthma. Chest 1991; 100: 1.254-1250.

11. Nowak D, J orres R, RabeKF et al. Salmeterol protects against hyperventilation-induced bronchoconstriction over 12 hours. Eur J Clin Pharmacol 1992; 43: 591-5.

12. Ullman A, Svedmyr N. Salmeterol, a new long acting inhaled $\beta_{2}$ adrenoceptor agonist: comparison with sal butamol in adult asthmatic patients. Thorax 1988; 43: 674-8

13. Lundback B, Rawlinson DW, Palmer J BD. On behalf of a European study group. Twelve month comparison of salmeterol and salbutamol as dry powder formulations in asthmatic patients. Thorax 1993; 48: 148-53.

14. Pearlman DS, Chervinsky $P$, L aforce $C$ et al. A comparison of salmeterol with albuterol in the treatment of mild-to-moderate asthma. N Engl J Med 1992; 327: 1.420-5.

15. National Health, Lung, and Blood Institute. National Institutes of Health. Guidelines for the Diagnosis and Management of Asthma. Bethesda, MD: 1991; № 91-3042.

16. Medical Graphics Corporation. Sistems Use's Manual. 1989, 1990, 1991.

17. Siegel S. Estadística no parametrica. México, Ed. Trillas, 1975.

18. Hollander M, Wolfe DA. Nonparametric statistical methods. New York, J ohn Wiley \& Sons, 1973.

19. Hetzel MR, Clark TJ . Comparison of normal and asthmatic circadian rhythms in peak expiratory flow rate. Thorax 1980; 35: 732-8.

20. Fitzpatrick MF, Mackay T, Driver $\mathrm{H}$ et al. Salmeterol in nocturnal asthma: a double bind, placebo controlled trial of a long acting inhaled $\beta_{2}$-agonist. $\mathrm{Br}$ Med J 1990; 301: 1.365-8.

21. Mackowiak JI, Lawrence BJ, Boyer J G et al. Effects of salmeterol on sleep scores using a quality of lifeinstrument in patients with mild/ moderateasthma. In ATS, San F rancisco, 1993; A 60.

22. Muir J F, Bertin L, Georges D. Salmeterol versus slow-release theophylline combined ketotifen in nocturnal: asthma: a Multicentre trial. Eur Respir J 1992; 5: 1.197-1200.

23. Dahl R, Earnshaw J S, Palmer J BD. Salmeterol; a four week study of a long-acting beta-adrenoceptor agonist for the treatment of reversibleairways disease. E ur Respir J 1991; 4: 1.178-84.

24. Cullum Valerie AJ B, Farmer D, J ack P et al. Salbutamol: a new selective adrenoceptive receptor stimulant. $\mathrm{Br} J$ Pharmacol 1969; 35: 141-51.

25. Crane J , Flatt A, J ackson $\mathrm{R}$ et al. Prescribed fenoterol and death from asthma in N ew Zealand. Lancet 1989; 29: 917-22. 